\title{
Ninjurin2, a Novel Homophilic Adhesion Molecule, Is Expressed in Mature Sensory and Enteric Neurons and Promotes Neurite Outgrowth
}

\author{
Toshiyuki Araki and Jeffrey Milbrandt \\ Division of Laboratory Medicine, Department of Pathology and Medicine, Washington University School of Medicine, St. \\ Louis, Missouri 63110
}

\begin{abstract}
A large number of cell adhesion molecules mediate cell-to-cell and cell-to-extracellular matrix interaction during development, differentiation and regeneration of the peripheral nervous system. Here, we report the identification of a novel cell surface adhesion molecule, ninjurin2 (for nerve injury induced protein 2). Ninjurin2 is a homolog of a homophilic cellular adhesion molecule, ninjurin1, that was previously isolated as a gene induced in Schwann cells after nerve injury. Ninjurin1 and 2 share conserved hydrophobic regions for their transmembrane domains; however, they do not contain comparable adhesion motifs nor do they interact with each other. In the peripheral nervous system, ninjurin2 is expressed constitutively in mature sensory and enteric neurons but not in glial cells or in auto-
\end{abstract}

nomic ganglia. Ninjurin2 is upregulated in Schwann cells surrounding the distal segment of injured nerve with a time course similar to that of ninjurin1, neural CAM, and L1. Ninjurin2 promotes neurite outgrowth from primary cultured dorsal root ganglion neurons, presumably via homophilic cellular interactions. Ninjurin2 is also highly expressed in hematopoietic and lymphatic tissues. Finally, the ninjurin2 gene is located on human chromosome 12p13 in which several disorders of unknown etiology have been mapped, including inflammatory bowel disease and acrocallosal syndrome.

Key words: cell adhesion; postmitotic; peripheral nervous system; hematopoietic and lymphatic organs; chromosome 12p13; inflammatory bowel disease; acrocallosal syndrome
Cell surface adhesion molecules play an important role in the peripheral nervous system (PNS) (Colello and Pott, 1997; Fu and Gordon, 1997). The adhesion molecules expressed in Schwann cells are primarily divided into two categories (for review, see Scherer, 1997). The first group includes proteins that characterize the nonmyelinating Schwann cell phenotype, including L1/nerve growth factor-inducible large external/neuron-glia cell adhesion molecule and neural cell adhesion molecule (N-CAM). These molecules are expressed throughout development in nonmyelinating Schwann cells. Conversely, Schwann cells of the myelinating lineage express these proteins at the promyelinating stage (i.e., before they start to myelinate axons). Previous reports have suggested that these proteins promote axonal growth and/or guide growing axons. The second group consists of "myelin proteins" whose expression is tightly linked to the myelinating Schwann cell phenotype. These include P0, peripheral myelin protein 22, and myelin-associated glycoprotein. Expression of these proteins begins soon after Schwann cells of the myelinating lineage start ensheathing axons. These myelin proteins are thought to be important for formation and maintenance of the myelin sheath in which a tight spiral of myelin membrane is formed.

During efforts to identify genes upregulated in Schwann cells after sciatic nerve axotomy, we identified a novel cell adhesion

Received July 26, 1999; revised Oct. 8, 1999; accepted Oct. 12, 1999.

This work was supported by grants from McDonnell Center for Cellular and Molecular Neurobiology and Uehara Memorial Foundation, and National Institute of Health Grant 5P01CA49712. We thank members of the laboratory for comments on this manuscript.

Correspondence should be addressed to Jeffrey Milbrandt, Division of Laboratory Medicine, Department of Pathology and Medicine, Washington University School of Medicine, 660 South Euclid Avenue, Box 8118, St. Louis, MO 63110. E-mail: jeff@milbrandt.wustl.edu.

Copyright (C) 1999 Society for Neuroscience 0270-6474/99/200187-09\$15.00/0 molecule, which we termed ninjurin1 (for nerve injury induced protein 1) (Araki and Milbrandt, 1996). Functional analysis revealed that ninjurin1 acts as a homophilic cellular adhesion molecule via a novel adhesion motif located in its N-terminal extracellular domain (Araki et al., 1997). In the PNS, basal expression of ninjurin1 is very low in adult sciatic nerve and DRG neurons, but it is highly upregulated in these tissues after nerve injury. Neurite extension of DRG neurons, which express ninjurin1, is increased when they are cultured on ninjurin1 substrata. These data suggested that, after peripheral nerve injury, ninjurin1 facilitates axonal regeneration by helping promote axonal extension via homophilic interactions between neuronal axons and Schwann cells.

Here, we report the identification of a new molecule bearing significant homology to ninjurin1, which we named ninjurin2. Ninjurin2 is also a cell surface adhesion molecule, but it does not share the adhesion motif sequence with ninjurin1. Expression of ninjurin 2 is constitutive and abundant in almost all the sensory ganglion neurons and enteric neurons but weak in peripheral glial cells and neurons in autonomic system. A developmental profile of ninjurin2 expression in DRG and enteric ganglia indicated that ninjurin2 expression is dramatically elevated in differentiated postmitotic neurons. In the CNS, ninjurin 2 expression is observed in radial glial cells but not in neurons. Ninjurin2 is upregulated in Schwann cells in the distal nerve segment after peripheral nerve injury, and it promotes neurite outgrowth from DRG neurons via ninjurin2-mediated homophilic cellular interaction.

\section{MATERIALS AND METHODS}

Cloning and sequence analysis. All sequencing analysis was performed on an Applied Biosystems (Foster City, CA) 373DNA sequencer using Taq DyeDeoxy Terminator cycle sequencing kits (Applied Biosystems). Se- 
quence editing, mapping, alignment, and contig generation were performed using the DNAstar software package. Expressed sequence tags (ESTs) were obtained from the Washington University-Merck EST project and sequenced completely. Based on the identified human ninjurin2 cDNA sequence, primers for mouse ninjurin2 (5'-ATGCTGGACGTGGCGCTCTTTATG-3' and 5'-TATGAAGACCAAGATGGTGGCAGCATT-3') were synthesized, and partial mouse ninjurin2 sequence was amplified by PCR. Rapid amplification of cDNA ends (RACE) PCR was performed using Klentaq-LA (Barnes, 1994) and human and mouse brain cDNA libraries, per the manufacturer's instructions (Clontech, Cambridge, UK). Products were cloned into the EcoRV site of pBluescript (Stratagene, La Jolla, CA), and full-length human and mouse cDNA sequences were determined. Putative transmembrane domains were predicted by PSORT algorithm (Nakai and Kanehisa, 1992).

For the ninjurin2 expression construct (pNINJ2), the coding region of the human ninjurin2 cDNA was amplified by Klentaq-LA from the brain marathon RACE cDNA library, using primers 5'-GTCGAGATCTACCATGGAATCAGCAAGAGAA-3' and 5'-CTAAAAGCTTAGAGAGGATTCCTTGAGGC-3'. The product was cloned into pCB6 expression vector (Brewer, 1994). Ninjurin1 expression construct ( $\mathrm{pN}$ INJ1) was described previously (Araki and Milbrandt, 1996). The human ninjurin2 genomic clone was obtained from a PAC genomic library (Roswell Park Cancer Center, Buffalo, NY).

Analysis of RNA expression. The human RNA master blot (Clontech) containing normalized samples of poly $\left(\mathrm{A}^{+}\right)$RNA was used according to the manufacturer's instructions. The blot was probed with random hexamer-primed ${ }^{32} \mathrm{P}$-labeled cDNA probe (nucleotides $90-888$ of human ninjurin2), and the signals were visualized with a PhosphorImager (Molecular Dynamics, Sunnyvale, CA). Human embryonic RNAs on the blot were pooled from fetal tissues of 10-30 weeks of gestation.

Sciatic nerves were transected as described previously (Araki and Milbrandt, 1996). All surgical procedures followed the National Institutes of Health guidelines for care and use of laboratory animals at Washington University. Northern analysis was performed as described previously (Araki and Milbrandt, 1996) using a ${ }^{32} \mathrm{P}$-labeled mouse ninjurin2 cDNA fragment.

Immunohistochemical analysis. A mixture of three synthetic peptides (see Fig. 5) was conjugated to keyhole limpet hemocyanin by glutaraldehyde cross-linking. The conjugated protein was used to immunize rabbits following standard procedures. Anti-ninjurin2 antibodies were purified by chromatography over an affinity column in which the peptides were linked to EAH Sepharose 4B (Amersham Pharmacia Biotech, Uppsala, Sweden) per the manufacturer's instructions. Protein blot analysis was performed as described previously (Lee et al., 1995). Immunohistochemical analysis of rat and mouse tissues was performed on $10 \mu \mathrm{m}$ sections of $4 \%$ paraformaldehyde-fixed tissues using standard methods. The purified antibodies were used at a 1:2500 dilution. Specific signal was amplified by Tyramide signal amplification system (Amersham Pharmacia Biotech) per the manufacturer's instructions and detected with indocarbocyanine (Cy3)-conjugated streptoavidin (Jackson ImmunoResearch, West Grove, PA). To identify proliferating cells, the bromodeoxyuridine (BrdU) labeling and detection kit (Boehringer Mannheim, Indianapolis, IN) was used per the manufacturer's protocol on tissue samples collected $1 \mathrm{hr}$ after intraperitoneal injection of BrdU labeling reagent. For immunocytochemistry of Chinese hamster ovary (CHO) cells, the purified antibodies were used at 1:2500 on $4 \%$ paraformaldehyde-PBS-fixed cells. For live cell staining, antibody was applied to ninjurin2-transfected $\mathrm{CHO}$ cells at $4^{\circ} \mathrm{C}$ for $30 \mathrm{~min}$, followed by fixation in $4 \%$ paraformaldehyde-PBS. Immunoreactivity was visualized using Cy3-conjugated anti-rabbit IgG (Jackson ImmunoResearch).

Cell culture and transfection. $\mathrm{CHO}$ and Jurkat cells were cultured as described previously (Araki and Milbrandt, 1996). The pCB6 or pNINJ2 plasmids were transfected into $\mathrm{CHO}$ cells via calcium phosphate precipitation and into Jurkat cells by electroporation. Stable transfectants were selected by growth in medium containing G418 $(400 \mu \mathrm{g} / \mathrm{ml}$ for $\mathrm{CHO}$ cells, $1 \mathrm{mg} / \mathrm{ml}$ for Jurkat cells), and individual clones were isolated by limiting dilution. Primary cultures of neurons from DRGs were prepared from embryonic day 16 (E16) rat embryos as described previously (Araki and Milbrandt, 1996). Dissociated neurons were plated on confluent monolayers of either control $\mathrm{CHO}$ cells or $\mathrm{CHO}$ cells expressing ninjurin2 in 12-well plates. Cultures were grown in a medium containing 90\% Eagle's minimal essential medium (Life Technologies, Gaithersburg, MD), $10 \%$ fetal calf serum, and $50 \mathrm{ng} / \mathrm{ml}$ mouse NGF. Six hours after plating, the cells were fixed with $4 \%$ paraformaldehyde in PBS, and immunohistochemistry was performed using anti-neurofilament $\mathrm{H}$ anti- bodies (Sigma, St. Louis, MO). Following previously established criteria (Gennarini et al., 1991; Araki and Milbrandt, 1996), 50 neurites were chosen for measurement. The length of each neurite was measured using Sigma Scan Pro 3.0, and statistical analysis was performed using Sigma Plot 3.0.

Peptide preparation. Three synthetic peptides with sequences corresponding to the indicated regions of human ninjurin2 (see Fig. $4 C$ ) were made and purified by HPLC. The purity and composition of the purified peptides were verified by mass spectrometry. The purified peptides were dissolved at $10 \mathrm{mg} / \mathrm{ml}$ in distilled water and stored at $-70^{\circ} \mathrm{C}$. A 30 residue synthetic peptide that was previously used for characterizing ninjurin1-mediated adhesion (P4 in Araki et al., 1997) was used as a negative control for aggregation assays.

Cell adhesion assays. Aggregation assays were performed using Jurkat cells stably transfected with pCB6 or pNINJ2 as described previously (Rothlein and Springer, 1986; Araki and Milbrandt, 1996). The formation of aggregates was monitored after $1 \mathrm{hr}$.

For heterophilic adhesion analysis, ninjurin1-expressing Jurkat cells (N1 cells) or wild-type Jurkat cells were stained green with $1 \mu \mathrm{M}$ 5-chloromethylfluorescein diacetate (CMFTA) (Molecular Probes, Eugene, OR), and ninjurin2-expressing cells (N2 cells) were stained red by $1 \mu \mathrm{M}$ 5-(and6)-(((4-chloromethyl)benzoyl)amino)tetramethylrhodamine (CMTMR) as described in the manufacturer's instructions (Molecular Probes). The cells were resuspended to $1 \times 10^{6}$ cells $/ \mathrm{ml}$, and $2 \mathrm{ml}$ of the mixed cell suspension were allowed to form aggregates in six-well culture plates for $2 \mathrm{hr}$. Quantitative analysis of heterophilic aggregation was performed as described previously with minor modifications (MurphyErdosh et al., 1995). In brief, the number of red cells and green cells in each aggregate was counted under an inverted fluorescent microscope (TE300; Nikon, Tokyo, Japan), and the percentage of N2 cell-dominant aggregates was calculated from two independent experiments $(50 \mathrm{~N} 2-$ predominant aggregates were counted in each experiment). Only aggregates with $>10$ cells were counted. When aggregates were attached to one another, they were counted as separate aggregates if they were bigger than 10 cells. If one of the attached aggregates was smaller than 10 cells, then the two aggregates were counted as a single aggregate. When the same number of CMTMR-labeled N2 cells and CMFTA-labeled N2 cells were mixed together, the percentage of single color cell population in aggregates was $50 \pm 4 \%$.

\section{RESULTS}

\section{Identification, sequence analysis, and genomic localization of ninjurin2}

Ninjurin2 was identified by performing a basic local alignment search tool (BLAST version 2.0) (Altschul et al., 1990) search of the dbEST database using the full-length human ninjurin1 cDNA sequence as a query. An EST clone (GenBank accession number H91351) that showed partial similarity with ninjurin1 was obtained from the Washington University-Merck EST project and sequenced. The $5^{\prime}$ end of the cDNA was obtained by RACE - PCR using a human brain Marathon RACE library as a template. The mouse ninjurin 2 cDNA was subsequently obtained by PCR amplification using primers corresponding to the human ninjurin2 cDNA.

The nucleotide sequence of the human ninjurin 2 cDNA predicts an open reading frame of 142 amino acids, which is $73 \%$ identical to mouse ninjurin2. Human ninjurin2 protein is $55 \%$ identical to human ninjurin1 but has no significant homology to any other known proteins in the database. Ninjurin2 has two hydrophobic regions, both of which can form transmembrane domains (PSORT algorithm; Nakai and Kanehisa, 1992). These transmembrane domains are the most highly conserved regions between ninjurin 1 and 2 . Ninjurin 2 does not contain any $\mathrm{N}$-linked glycosylation sites or a signal sequence. Residues 26-37 of ninjurin1, which contain the homophilic adhesive motif (Araki and Milbrandt, 1996), is not conserved in ninjurin2. Overall, these features suggest that ninjurin2 is a membrane protein like ninjurin1, but that it is functionally distinct from ninjurin1.

To analyze the genomic locus of ninjurin2, we identified and 
A

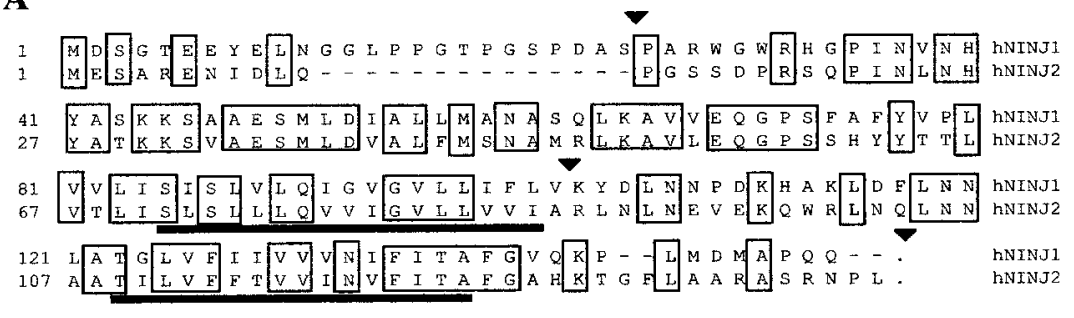

$\mathbf{B}$

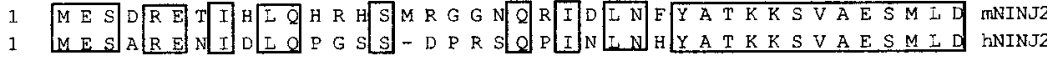

41 VALFMSNAMRLRS

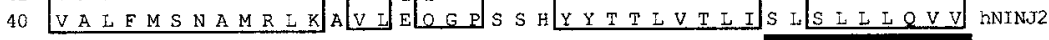

81 T L L LV F I A I I N L N E V EN O R H L N K L N N A A T I LVF I T V V I N mNINJ2

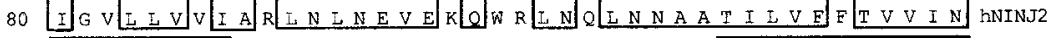

121 I FITAFGA H A A S MARTSSNQI .

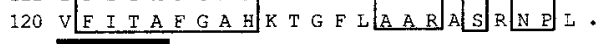

mNINJ2

hNINJ2

sequenced a human ninjurin2 genomic clone. The ninjurin2 gene has three introns, and the location of all three of these introns is precisely conserved between ninjurin1 and ninjurin2 (Fig. 1). A BLAST search against the high throughput genome sequence database revealed that human ninjurin2 is located on chromosome 12, region p13. A search of the On-line Mendelian Inheritance in Men (OMIM) database showed that some human diseases of unknown pathogenesis have been linked to this region, including acrocallosal syndrome (polydactyly and loss of corpus callosum) and inflammatory bowel disease.

\section{Ninjurin2 is located on the plasma membrane and mediates homophilic adhesion}

To characterize the ninjurin2 protein and examine its cellular localization, we generated polyclonal antiserum against a mixture of three synthetic peptides derived from the ninjurin $2 \mathrm{~N}$ terminus (amino acids 1-30, 15-45, and 31-60, as shown in Fig. 4C). Anti-ninjurin 2 antibodies were then purified by immunoaffinity chromatography. These antibodies recognized a $\sim 20 \mathrm{kDa}$ protein expressed in $\mathrm{CHO}$ cells that were stably transfected with pNINJ2 but that was absent in either control $\mathrm{CHO}$ cells or $\mathrm{CHO}$ cells stably expressing ninjurin1 (Fig. 2).

The high sequence homology with ninjurin1 in the putative transmembrane domain and immunocytochemical analysis of $\mathrm{CHO}$ cells stably expressing ninjurin2 suggested that ninjurin2 is located on the cytoplasmic membrane (Fig. $3 B$ ). To examine this possibility, we performed immunostaining of live $\mathrm{CHO}$ cells stably expressing ninjurin 2 using conditions in which antibodies do not penetrate the cell membrane and are not internalized. Antininjurin2 antibodies showed intense staining on the cell surface (Fig. $3 C$ ), whereas negative control $\beta$-actin antibodies showed no staining under similar conditions (data not shown). This clearly indicated that ninjurin 2 is located on the plasma membrane and that the $\mathrm{N}$-terminal region of ninjurin2 (to which the antibodies were raised) is located extracellularly.

The cell surface localization of ninjurin 2 and its homology with the adhesion molecule ninjurin1 suggested that ninjurin2 might also be an adhesion molecule. To test whether ninjurin 2 mediates cellular adhesion, we performed standard cell aggregation assays using Jurkat cells (Shimizu et al., 1990). Jurkat cells were stably transfected with either pNINJ2 or pCB6 (nonrecombinant vector). Expression of ninjurin2 in the Jurkat cells transfected with
Figure 1. Sequence analysis of ninjurin2. A, Alignment of human ninjurin1 and ninjurin2. Identical residues are boxed, and putative transmembrane domains are underlined. Arrowheads denote intron-exon junctions. Note that these sites are conserved between the ninjurin1 and 2 genes. $B$, Alignment of human and mouse ninjurin 2 amino acid sequences. Identical residues are boxed, and putative transmembrane domains are underlined.

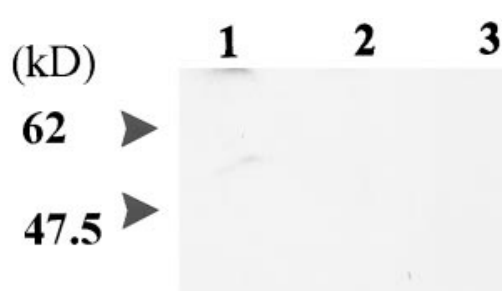

\section{5 \\ 25}

\section{5}

Figure 2. Protein blot analysis of ninjurin2. Proteins from lysates prepared from native $\mathrm{CHO}$ cells (lane 1), $\mathrm{CHO}$ cells stably transfected with a pNINJ1 expression construct (lane 2), and CHO cells stably transfected with pNINJ2 construct (lane 3) were electrophoresed on $12 \%$ SDSpolyacrylamide gels, transferred to nitrocellulose, and incubated with affinity-purified anti-ninjurin 2 antibodies. Ninjurin2 was visualized by using enhanced chemiluminescence.

pNINJ2 (N2 cells) but not in pCB6 was confirmed by immunoblot analysis (data not shown). Jurkat cells stably expressing ninjurin2 demonstrated increased aggregation compared with cells transfected with pCB6 (Fig. 4A,B). Increased aggregation of ninjurin2-expressing Jurkat cells (N2 cells) was quantified in aggregation assays in 96-well culture plates. After $1 \mathrm{hr}$, the percentage of cells in aggregates was $83 \pm 4 \%$ in N2 cells and $14 \pm$ $4 \%$ in control cells. To identify which region of ninjurin 2 is responsible for ninjurin2-mediated cell adhesion, we tested three partially overlapping peptides corresponding to the $\mathrm{N}$-terminal extracellular region of ninjurin2 for their ability to inhibit ninjurin2-mediated cellular aggregation (Fig. 4C,D). Peptide (P) 1 and $\mathrm{P} 2$ inhibited aggregation in a dose-dependent manner, with 

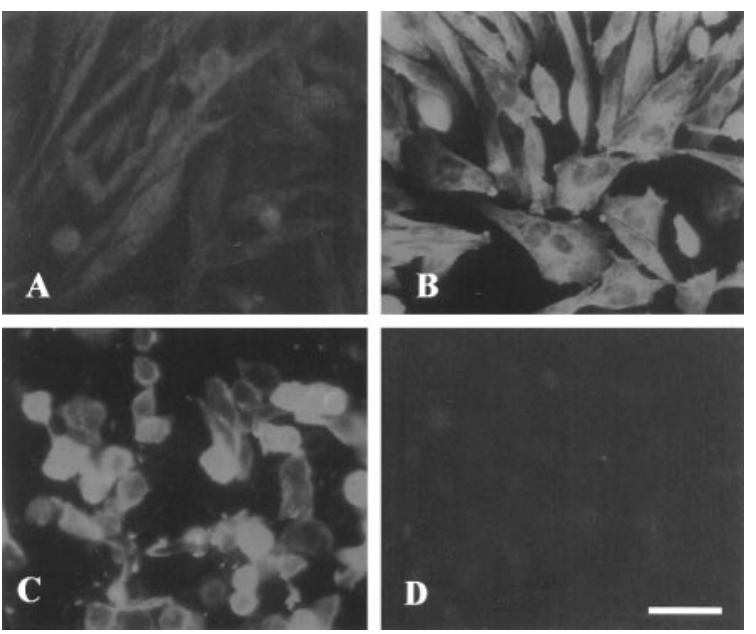

Figure 3. Ninjurin2 is localized on the plasma membrane. Immunocytochemical localization of ninjurin2 on wild-type $\mathrm{CHO}$ cells $(A)$ and $\mathrm{CHO}$ cells transfected with pNINJ2 $(B-D)$. Anti-ninjurin2 antibodies were applied to cells either after fixation with $4 \%$ paraformaldehyde $(A$, $B$ ) or before fixation $(C)$. In $D$, anti-ninjurin2 antisera were blocked by incubation with the peptide immunogen and applied to cells as in $B$ (see Materials and Methods). Scale bar, $40 \mu \mathrm{m}$.

P2 more effectively inhibiting aggregation than P1. P3 was less effective than $\mathrm{P} 1$ and $\mathrm{P} 2$ at low concentrations. It could not be tested at higher concentrations because of the high viscosity of the solution. These results indicated that the aggregation observed in ninjurin2-expressing cells is attributable to ninjurin2mediated interactions and that the $\mathrm{N}$-terminal 45 residues of ninjurin2 contain the functional adhesion domain.

The ability of ninjurin 2 to associate heterophilically with ninjurin1 or other molecules expressed on wild-type Jurkat cells was also investigated, because many other homophilic adhesion molecules associate heterophilically with their family members. Aggregation assays were performed by mixing an equal number of Ninjurin1-expressing Jurkat cells (N1 cells) or wild-type cells (stained green) and N2 cells (stained red). For quantitative analysis, the number of green cells and red cells in each aggregate was counted. Aggregates formed in the N1-N2 mixture were either $\mathrm{N} 1$ cell-predominant or N2 cell-predominant (Fig. $4 E$ ), whereas mixed aggregates (containing $\mathrm{N} 1$ and $\mathrm{N} 2$ cells) were very rare. Only N2-predominant aggregates were observed in the N2 wildtype mixture (Fig. $4 F$ ). The percentage of N2 cells in N2 cellpredominant aggregates was calculated for each of the mixtures (Fig. $4 G$ ). The percentages of N2 cells were not significantly different, regardless of the cell type with which they were mixed, indicating that $\mathrm{N} 2$ cells do not interact with either wild-type or N1 cells. These results indicate that ninjurin2 does not show heterophilic association with ninjurin 1 or surface molecules expressed on wild-type Jurkat cells.

\section{Expression of ninjurin2 mRNA and protein}

To obtain further insight into the function of ninjurin 2 in vivo, we examined mRNA expression in a variety of embryonic and adult organs on a human tissue mRNA blot. High expression of ninjurin2 mRNA was observed in a limited number of organs. In the adult, ninjurin 2 mRNA expression was highest in the bone marrow, followed by peripheral leukocytes, lung, and lymph nodes (Fig. 5).

We further examined the expression of ninjurin2 in adult rat organs by immunohistochemistry. In addition to the organs ex- pressing ninjurin 2 identified by RNA blots, other tissues showed expression of ninjurin 2 with restricted distribution patterns. In the kidney, ninjurin2 was detected specifically in the glomeruli (Fig. 6A). In the adrenal gland, ninjurin 2 expression was observed only in the glomerular layer of the cortex (Fig. 6B). In the CNS, neuronal expression of ninjurin2 was very low; however, ninjurin2 was detected in radial glial cells during development and in adulthood (data not shown).

\section{Ninjurin2 is expressed in mature postmitotic sensory and enteric neurons}

To determine whether ninjurin2 plays a role in the PNS, expression of ninjurin 2 was examined in detail by immunohistochemistry. Expression of ninjurin2 was detected in most neurons of the sensory and enteric ganglia, but in contrast to ninjurin1 (Araki and Milbrandt, 1996), ninjurin2 was not expressed in the supportive glial cells. Ninjurin2 expression was intense in the adult rat trigeminal (Fig. 6C), DRG (Fig. 6D), and nodose ganglia (data not shown). The intensity of ninjurin2 immunoreactivity was similar in most neurons and did not correlate with neuronal size. In the enteric nervous system, ninjurin 2 was detected in neurons of both the submucosal and myenteric plexuses (Fig. 6F). In contrast, ninjurin2 expression in the superior cervical ganglion (Fig. 6E) and parasympathetic ganglion in the salivary gland (data not shown) was very low.

To explore the developmental regulation of ninjurin2 expression in the PNS, immunohistochemical analysis was performed on mouse DRG at E14, E19, and postnatal day 2 (P2) and in the mouse enteric ganglia at E17, P1, and P3. In the DRG, ninjurin2 expression was very weak at E14 (Fig. 7A), became apparent at E19 (Fig. 7B), and by P2, the intensity of staining was comparable with the adult level (Fig. $7 C$ ). An examination of the enteric ganglia revealed that ninjurin2 is not expressed at E17 (Fig. 7D), but weak ninjurin2 immunoreactivity was observed in neurons of the myenteric ganglia at P1 (Fig. $7 E$ ) and was more intense at P3 (Fig. $7 F$ ). In contrast, ninjurin2 expression was undetected in the submucosal plexus.

Neuronal proliferation in the myenteric ganglia is almost complete by P3, whereas in the submucosal plexus, neurons are generated later in development (by P14) (Pham et al., 1991). This raised the possibility that ninjurin2 is expressed only in postmitotic enteric neurons. To test this hypothesis, BrdU was injected into 3-d-old mice. Animals were killed $1 \mathrm{hr}$ later, and gut sections were examined immunohistochemically to assess ninjurin2 expression and BrdU incorporation. We found that ninjurin2immunoreactive cells in the myenteric plexus lacked BrdU incorporation (Fig. 8C,D), whereas some neuron-specific enolase (NSE)-positive cells in the submucosal plexus were BrdU-positive (Fig. $8 E, F$ ). These results suggest that ninjurin2 expression occurs after enteric neurons become postmitotic.

\section{Ninjurin2 is upregulated after nerve injury in Schwann cells}

We were interested in characterizing the role of ninjurin2 after nerve injury, because the related ninjurin1 is highly induced in Schwann cells after nerve injury. To examine the expression of ninjurin2 after nerve injury, immunohistochemistry was performed to detect ninjurin2 in normal and injured nerves. In normal sciatic nerve, ninjurin2 expression was weak (Fig. 9A), but it was greatly upregulated $7 \mathrm{~d}$ after nerve transection in the distal segment of the injured nerve (Fig. 9B). The interdigitating pattern of ninjurin2 immunoreactivity in the injured nerve (Fig. 9D) 

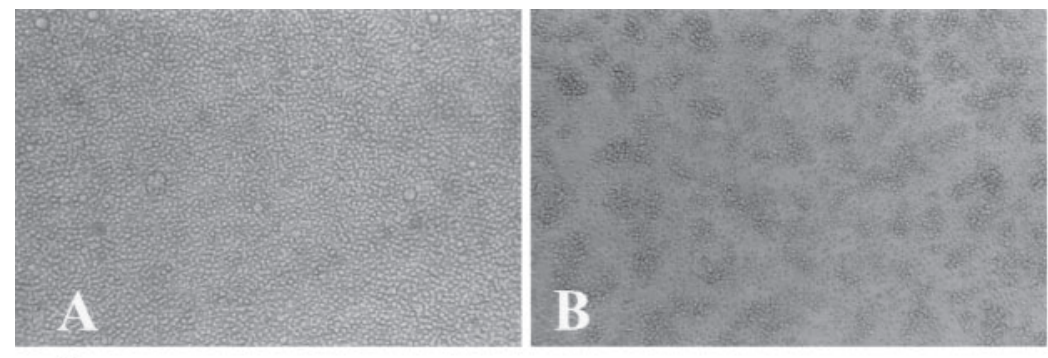

C

10 MESARENIDLQPGSSDPRSQPINLNHYATK (P1) 20

40

DPRSQPINLNHYATKKSVAESMLDVALFMS (P2)

KSVAESMLDVALFMSNAMRLKAVLEQGPSS (P3)
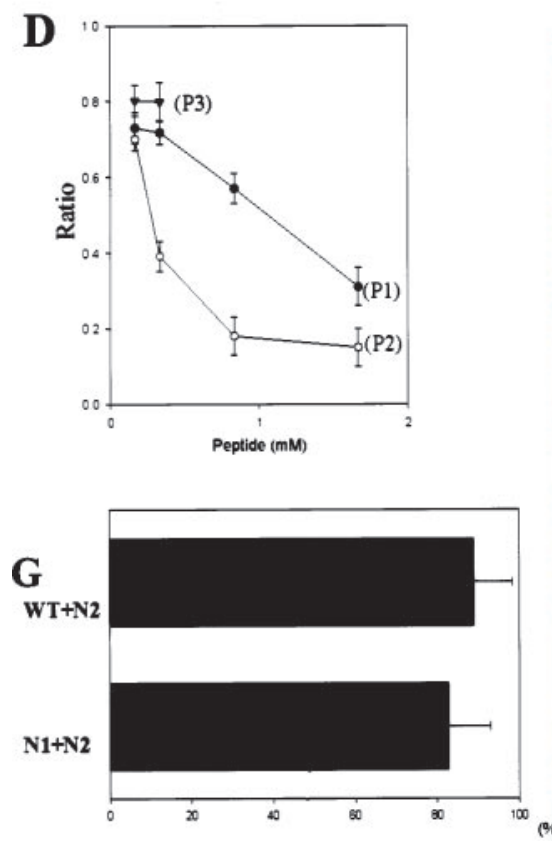
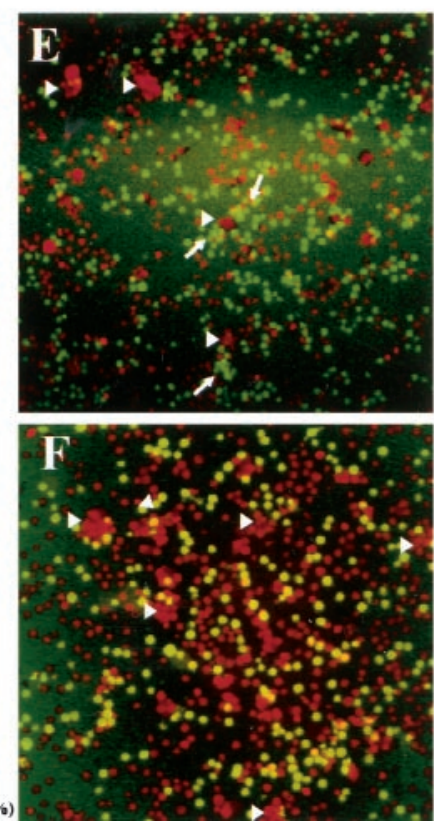

Figure 4. Ninjurin2 mediates homophilic cell adhesion. Native Jurkat cells $(A)$ and Jurkat cells stably transfected with pNINJ2 $(B)$ were resuspended at $1 \times 10^{6}$ cells $/ \mathrm{ml}$ and allowed to aggregate at $37^{\circ} \mathrm{C}$ for $1 \mathrm{hr}$. Note the presence of large aggregates in $B$. $C$, List of peptides used in competition experiments and also as antigens to raise anti-ninjurin2 antisera. D, Aggregation assays were performed using ninjurin2 stably expressing Jurkat cells in the presence of each of the indicated peptides. The number of cells in aggregates was determined after $1 \mathrm{hr}$. The ratio of cells in aggregates to total cells was calculated and plotted versus peptide concentration. Data represents the mean \pm SD of three independent experiments. $E, F$, Aggregation assays were performed with a mixture of $\mathrm{N} 2$ cells (stained with red-orange fluorescent dye) and either N1 cells $(E)$ or wild-type cells $(F)$ (stained with green fluorescent dye). Note that the green cells indicated by arrows and red-orange cells indicated by arrowheads individually form aggregates in $E$, and only red cells form aggregates in $F$ as indicated by arrowheads. $G$, Quantitative analysis of aggregation assays using mixed cell populations. The aggregation assays were performed as in $E$ and $F$, and red cells and green cells in each aggregate were counted. The graph represents the mean \pm SD percentage of $\mathrm{N} 2$ cells in aggregates that consist predominantly of $\mathrm{N} 2$ cells. closely resembled that observed for ninjurin1 (Fig. 9C), indicating that ninjurin2 is expressed by Schwann cells.

To further characterize the regulation of ninjurin2 expression after nerve injury, we examined ninjurin 2 mRNA levels for up to 8 weeks after nerve injury (Fig. $9 E$ ). A $1.0 \mathrm{~kb}$ ninjurin 2 mRNA was detected at low levels in normal nerve but was highly upregulated after nerve injury and reached peak levels 7-14 d after injury. This time course of expression is similar to what has been observed for other nonmyelinating Schwann cell marker molecules, including ninjurin1 and p75 (Taniuchi et al., 1988; Araki and Milbrandt, 1996). The message level remained high for up to $56 \mathrm{~d}$ when the nerve was completely transected, but when the injury was partial and nerve regeneration could occur, ninjurin2 mRNA expression returned to low levels after 28-56 d (data not shown).

\section{Ninjurin promotes neurite outgrowth from primary cultured DRG neurons}

Upregulation of ninjurin2 in Schwann cells after nerve injury suggested that it may promote nerve regeneration by homophilic adhesive interactions as has been observed with other adhesion molecules (Seilheimer and Schachner, 1988; Lemmon et al.,
1989). To test this possibility, we examined neurite outgrowth in DRG neuron-CHO cell cocultures (Eichler and Rich, 1989). DRG neurons were dissected from E16 rat embryos, and dissociated neurons were seeded at low density onto confluent monolayer of either wild-type $\mathrm{CHO}$ cells or $\mathrm{CHO}$ cells stably expressing ninjurin2. The culture was fixed after $6 \mathrm{hr}$ of culture, and neurites were visualized by immunostaining of neurofilament $\mathrm{H}$. As described previously (Araki and Milbrandt, 1996), 50 neurons were randomly selected from each culture condition, and the length of the longest neurite per neuron, which did not have contacts with nearby neurons, was measured. As shown in Figure $9 F$, neurons plated on $\mathrm{CHO}$ cells expressing ninjurin 2 extended significantly longer neurites than the ones plated on wild-type CHO cells. These results indicate that ninjurin2, like ninjurin1, promotes neurite outgrowth from primary cultured neurons.

\section{DISCUSSION}

We have identified a novel member of the ninjurin family of adhesion molecules. Members of this family share high homology in the putative transmembrane domains, lack signal peptide sequences, and have the $\mathrm{N}$-terminal hydrophilic region located 
Figure 5. Expression of ninjurin 2 mRNA in human tissues. A human mRNA dot blot (Clontech) was probed with a ${ }^{32} \mathrm{P}$-labeled fragment of the human ninjurin2 cDNA. The hybridization signal intensity was quantified by using a PhosphorImager.

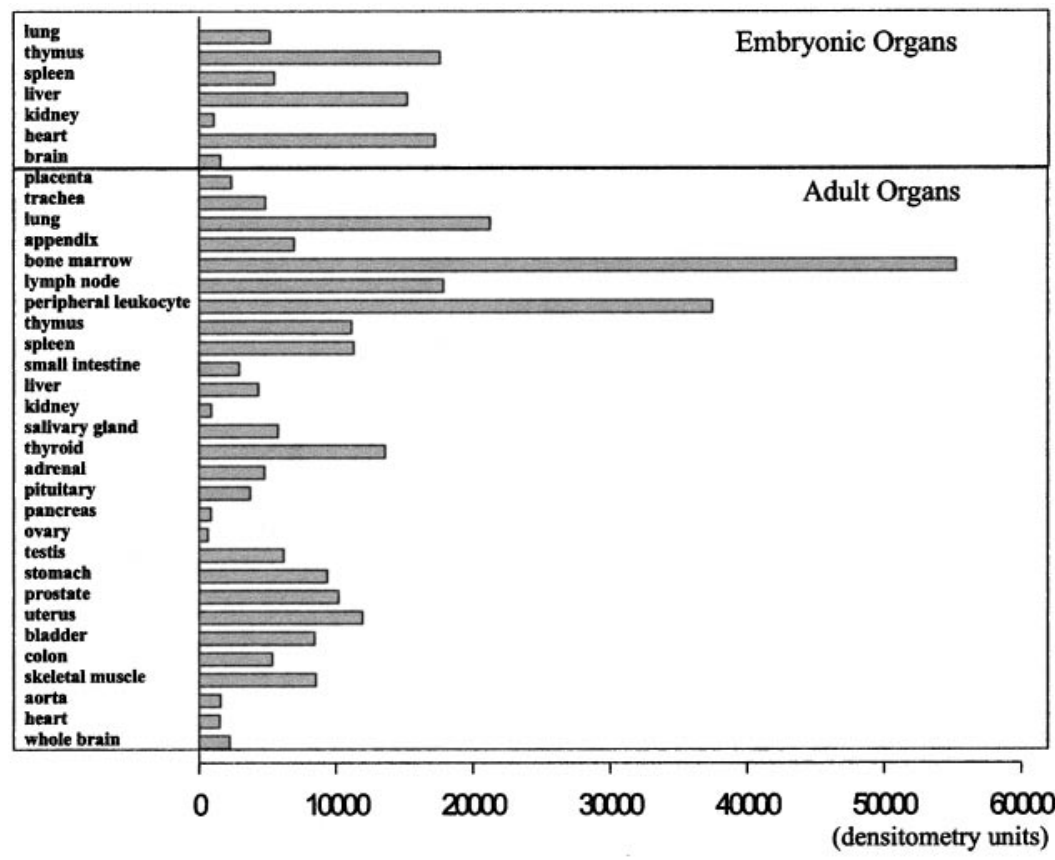

Figure 6. Immunohistochemical analysis of ninjurin2 expression in adult rat. Affinity-purified antibodies were used to detect ninjurin2 expression by immunohistochemistry in kidney $(A)$, adrenal $(B)$, trigeminal ganglion $(C)$, DRG $(D)$, superior cervical ganglion $(E)$, and enteric plexus $(F)$. Arrows denote glomeruli in $A$ and glomerular layer in the adrenal cortex in B. Arrows and arrowheads denote submucosal and myenteric plexus, respectively, in $F$. Scale bars, $100 \mu \mathrm{m}$.
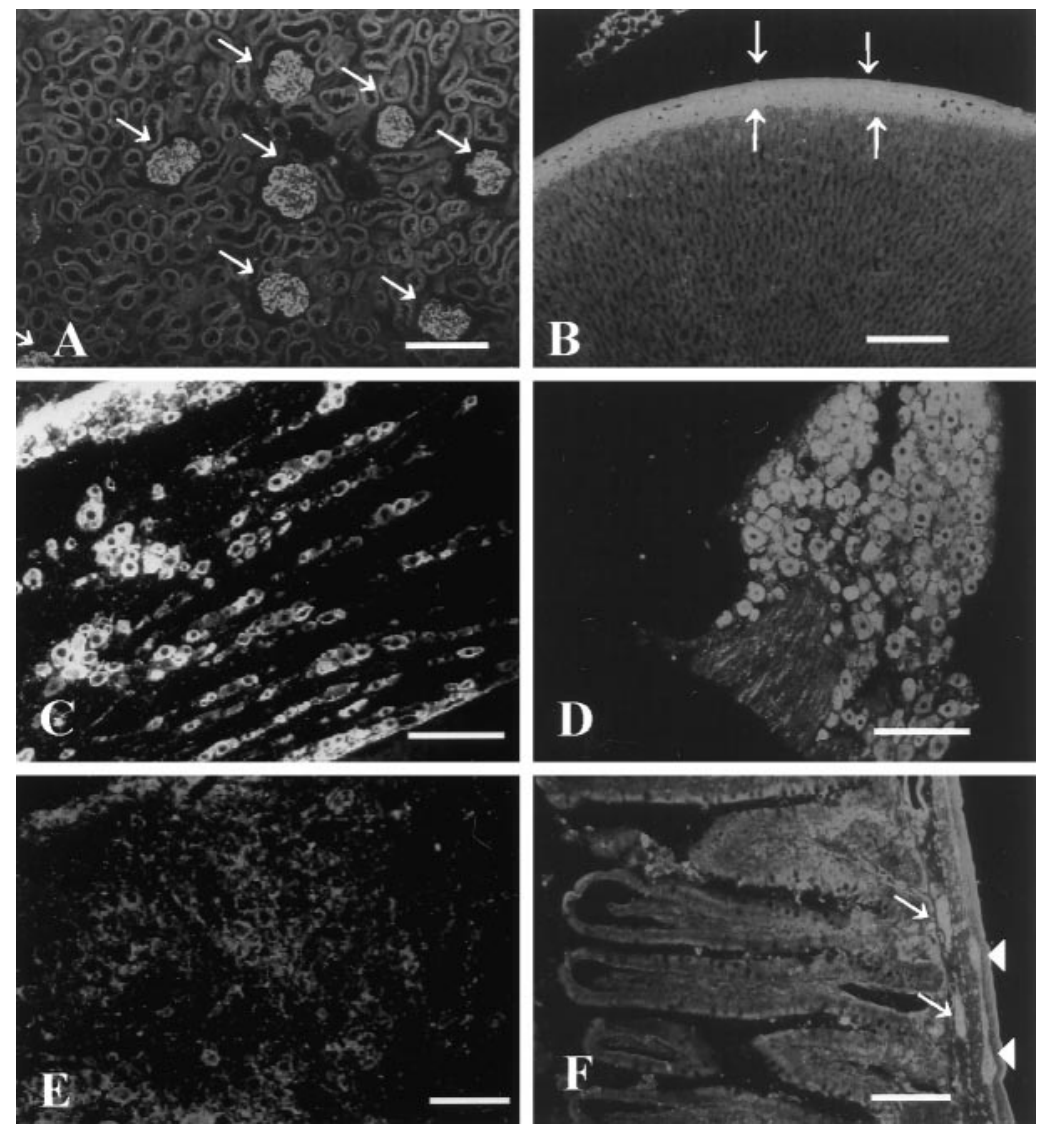

extracellularly. The extracellular regions, especially the domains involved in adhesive interactions, are diverse. Although interactions between family members are commonly observed with other adhesion molecules (Brummendorf and Rathjen, 1996), no heterophilic interactions were observed between ninjurin2 and ninjurin1. Consistent with these results, residues comprising the ninjurin1 adhesion motif are not conserved in the ninjurin2 adhesion motif located between residues 16 and 45 .

The tissue distribution of ninjurin proteins indicates that they are involved in multiple functional systems in the body like most other known adhesion molecules. Ninjurin1 showed a wide distribution primarily among organs of epithelial origin, whereas 

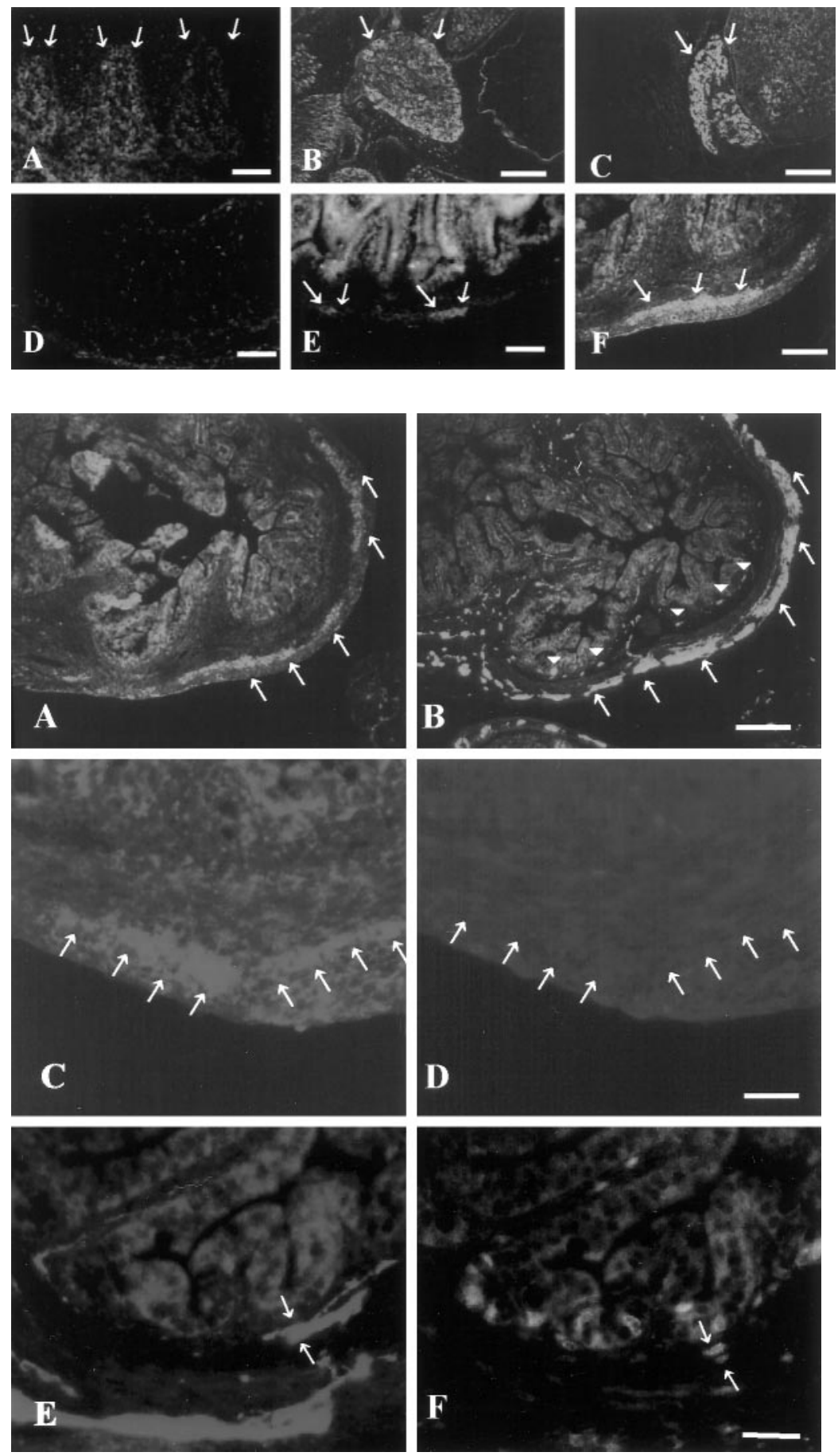

Figure 7. Expression of ninjurin2 in sensory and enteric neurons during development. Immunohistochemistry was used to detect ninjurin2 in mouse DRG at E14 $(A), \mathrm{E} 19(B)$, and $\mathrm{P} 3(C)$ and in mouse enteric plexus at $\mathrm{E} 17(D), \mathrm{P} 1(E)$, and $\mathrm{P} 3(F)$. Arrows denote the ganglia in each micrograph. Scale bars: $A, D, E, 50 \mu \mathrm{m} ; B, C, F, 150 \mu \mathrm{m}$.
Figure 8. Ninjurin2 is expressed in postmitotic neurons in enteric ganglia. $A, B$, Ninjurin2 immunoreactivity in enteric plexus in $\mathrm{P} 3$ mouse gut $(A)$ was compared with neuron-specific enolase immunoreactivity on an adjacent section $(B)$. Note that myenteric neurons denoted by arrows express ninjurin2, but submucosal neurons denoted by arrowheads in $B$, which differentiate later than myenteric neurons, lack ninjurin2 expression in $A$. Scale bar, $100 \mu \mathrm{m}$. $C-F, \mathrm{BrdU}$ and either ninjurin2 $(C, D)$ or NSE $(E, F)$ were visualized on the same section of mouse gut at P3. A mouse (P3) was injected with BrdU and killed $1 \mathrm{hr}$ later for immunohistochemistry. Ninjurin2 $(C)$ and NSE expression $(E)$ were visualized by Cy3conjugated secondary antibody, and proliferating cells were visualized by FITC-conjugated anti-BrdU immunohistochemistry $(D, F)$. The ninjurin2-positive myenteric ganglia lack BrdU staining (arrows in $C$ and $D$ ), whereas the NSE-positive cells in the submucosal ganglia indicated by arrows in $E$ and $F$ are BrdU-positive. Note that $C$ and $D$ represent the same section as do $E$ and $F$. Scale bars, $50 \mu \mathrm{m}$. ninjurin2 showed more restricted distribution; ninjurin2 was highly expressed in lymphatic and hematopoietic organs. In addition, the majority of human ninjuirn 2 cDNA sequence in the dbEST database is derived from "germinal center B cells" libraries (data not shown). This suggests that ninjurin2 expression in the lymphatic cells is involved in B lymphocyte function, perhaps during maturation of $\mathrm{B}$ lymphocytes to the antibody-producing cells.

Ninjurin2 expression in mature sensory and enteric neurons clearly distinguishes this molecule from ninjurin1 and other cell surface adhesion molecules. In mouse DRG, many neurons are generated between E10 and E13 in a neurotrophin-3-dependent manner (Farinas et al., 1996, 1998). In the enteric nervous system, submucosal neurons are generally born later than myenteric neurons with the peak of neuronal proliferation in myenteric plexus occurring before birth, whereas the majority of submucosal neurons continue to proliferate after birth (Pham et al., 1991). In each of these neuronal populations, the appearance of ninjurin2 immunoreactivity coincides with the age at which they differentiate into postmitotic neurons. These results could indicate that ninjurin2 expression is necessary for growth arrest of certain population of peripheral neurons or that ninjurin 2 expression is suppressed while neuronal precursors are stimulated by mitogenic signals. Other roles of ninjurin2 in the normal adult PNS may 

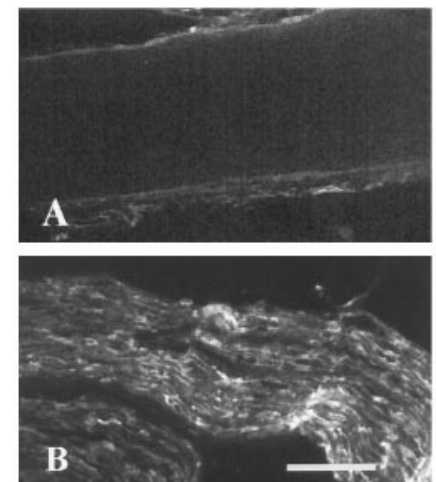
nerve injury. $A-D$, Immunohistochemistry was used to detect ninjurin 2 on a longitudinal section of the normal sciatic nerve $(A)$ or sciatic nerve segment distal to the site of injury (7 d after injury; $B$, low magnification; $D$, high magnification). In $C$, the section adjacent to $D$ was stained with antibodies to ninjurin1. The very similar expression patterns in $C$ and $D$ indicate that ninjurin2 is expressed in Schwann cells in injured nerve. Scale bars: $B, 100 \mu \mathrm{m} ; D, 50 \mu \mathrm{m}$. $E$, Total RNA $(10 \mu \mathrm{g})$ was isolated from sciatic nerves distal to the site of transection at the indicated times after axotomy. The samples were electrophoresed, blotted, and hybridized with a ${ }^{32}$ P-labeled ninjurin 2 cDNA probe. $F$, Ninjurin 2 promotes neurite outgrowth from primary cultured DRG neurons. DRG neurons from E16 rat embryos were seeded onto a confluent monolayer of control $\mathrm{CHO}$ cells or $\mathrm{CHO}$ cells expressing ninjurin2. Six hours later, the cells were fixed, and neurites were visualized by immunostaining with neurofilament $\mathrm{H}$ antibodies. Neurite length was quantified by measuring neurites from $\sim 50$ neurons grown under each condition in three independent experiments. Data represent mean \pm SD length.
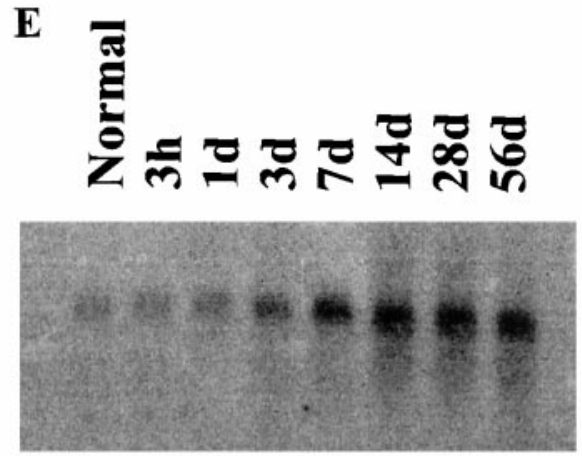

$\mathbf{F}$

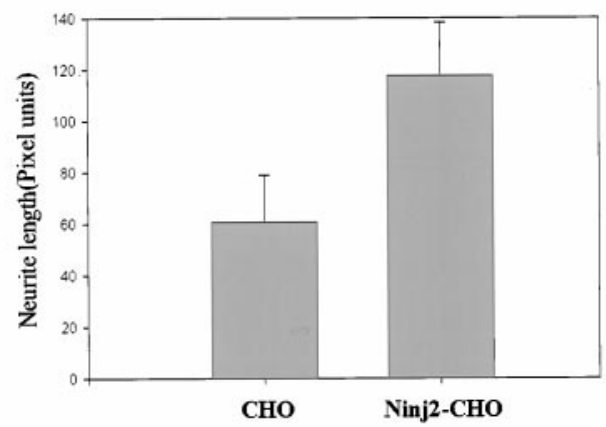

include interaction between neurons and peripheral sensory organs that express ninjurin2 or other unidentified heterophilic partners of ninjurin2.

Expression of ninjurin2 in the DRG neurons is not uniform; there are a very small number of neurons in which ninjurin2 immunoreactivity is weak or absent. Such neurons are more clearly observed after axonal injury when most DRG neurons increase ninjurin2 expression. Coimmunostaining of ninjurin1 and ninjurin 2 showed that ninjurin 1 is also not induced in neurons that lack ninjurin2 expression (data not shown). This result suggests that some DRG neurons are dependent on surface molecules other than ninjurins for axonal regeneration or other functions.

Although ninjurin1 and 2 are differentially expressed in the PNS, ninjurin2 is likely to play a role in nerve regeneration via homophilic interaction, just like ninjurin1. The time course of upregulation of ninjurin2 after nerve injury is similar to that of ninjurin1, and the level of neurite outgrowth enhancement in coculture experiments with DRG neurons and ninjurin2expressing $\mathrm{CHO}$ cells was comparable with the ninjurin1mediated effect (Araki and Milbrandt, 1996). During peripheral nerve regeneration, ninjurin 2 may have additional or synergistic effect on the neurite outgrowth-promoting effects of ninjurin1 and other adhesion molecules, including N-CAM and L1 (Bixby et al., 1988; Seilheimer and Schachner, 1988).

In the human chromosome region $12 \mathrm{p} 13$ in which ninjurin2 is located, several diseases of unknown etiology have been mapped (Pfeiffer et al., 1992; Satsangi et al., 1996; Duerr et al., 1998). Diseases with neurological defects, such as acrocallosal syndrome, are of particular interest because mutations in another adhesion molecule, L1, result in severe neurological defects, including MASA (mental retardation, aphagia, shuffling gate, and adducted thumbs) syndrome (Vits et al., 1994). Possible associa- tion of ninjurin2 with inflammatory bowel disease is also interesting from both immunological and neurological perspectives. From the immunological perspective, differences in lymphocyte homing capability between inflammatory bowel disease and normal intestine have been reported previously (Panes and Granger, 1998). Surface adhesion molecules like ninjurin 2 expressed on lymphocytes could be important for the pathogenesis of local and/or systemic inflammatory disorders. On the other hand, a recent report showed that moderate degeneration of myenteric neurons could cause severe inflammation in the gut (Bush et al., 1998). Thus, ninjurin 2 mutations could also affect gut physiology by altering enteric neuron activities, such as regulation of mucosal blood flow.

\section{REFERENCES}

Altschul SF, Gish W, Miller W, Myers EW, Lipman DJ (1990) Basic local alignment search tool. J Mol Biol 215:403-410.

Araki T, Milbrandt J (1996) Ninjurin, a novel adhesion molecule, is induced by nerve injury and promotes axonal growth. Neuron 17:353-361.

Araki T, Zimonjic DB, Popescu NC, Milbrandt J (1997) Mechanism of homophilic binding mediated by ninjurin, a novel widely expressed adhesion molecule. J Biol Chem 272:21373-21380.

Barnes WM (1994) PCR amplification of up to 35-kb DNA with high fidelity and high yield from lambda bacteriophage templates. Proc Natl Acad Sci USA 91:2216-2220.

Bixby JL, Lilien J, Reichardt LF (1988) Identification of the major proteins that promote neural process outgrowth on Schwann cells in vitro. J Cell Biol 107:353-361.

Brewer CB (1994) Cytomegalovirus plasmid vectors for permanent lines of polarized epithelial cells. Methods Cell Biol 43:233-245.

Brummendorf T, Rathjen FG (1996) Structure/function relationships of axon-associated adhesion receptors of the immunoglobulin superfamily. Curr Opin Neurobiol 6:584-593.

Bush TG, Savidge TC, Freeman TC, Cox HJ, Campbell EA, Mucke L, Johnson MH, Sofroniew MV (1998) Fulminant jejuno-ileitis following ablation of enteric glia in adult transgenic mice. Cell 93:189-201. 
Colello RJ, Pott U (1997) Signals that initiate myelination in the developing mammalian nervous system. Mol Neurobiol 15:83-100.

Duerr RH, Barmada MM, Zhang L, Davis S, Preston RA, Chensny LJ, Brown JL, Ehrlich GD, Weeks DE, Aston CE (1998) Linkage and association between inflammatory bowel disease and a locus on chromosome 12. Am J Hum Genet 63:95-100.

Eichler ME, Rich KM (1989) Death of sensory ganglion neurons after acute withdrawal of nerve growth factor in dissociated cell cultures. Brain Res 482:340-346.

Farinas I, Yoshida CK, Backus C, Reichardt LF (1996) Lack of neurotrophin-3 results in death of spinal sensory neurons and premature differentiation of their precursors. Neuron 17:1065-1078.

Farinas I, Wilkinson G, Backus C, Reichardt L, Patapoutian A (1998) Characterization of neurotrophin and Trk receptor functions in developing sensory ganglia: direct NT-3 activation of TrkB neurons in vivo. Neuron 21:325-334.

Fu SY, Gordon T (1997) The cellular and molecular basis of peripheral nerve regeneration. Mol Neurobiol 14:67-116.

Gennarini G, Durbec P, Boned A, Rougon G, Goridis C (1991) Transfected F3/F11 Neuronal cell surface protein mediates intercellular adhesion and promotes neurite outgrowth. Neuron 6:595-606.

Lee SL, Tourtellotte LC, Wesselschmidt RL, Milbrandt J (1995) Growth and differentiation proceeds normally in cells deficient in the immediate early gene NGFI-A. J Biol Chem 270:9971-9977.

Lemmon V, Farr KL, Lagenaur C (1989) L1-Mediated axon outgrowth occurs via a homophilic binding mechanism. Neuron 2:1597-1603.

Murphy-Erdosh C, Yoshida CK, Paradies N, Reichardt LF (1995) The cadherin-binding specificities of B-cadherin and LCAM. J Cell Biol 129:1379-1390.

Nakai K, Kanehisa M (1992) A knowledge base for predicting protein localization sites in eukaryotic cells. Genomics 25:897-911.

Panes L, Granger DN (1998) Leukocyte-endothelial cell interactions: molecular mechanisms and implications in gastrointestinal disease. Gastroenterology 114:1066-1090.

Pfeiffer RA, Legat G, Trautmann U (1992) Acrocallosal syndrome in a child with de novo inverted tandem duplication of 12p11.2-p13.3. Ann Genet 35:41-46.

Pham TD, Gershon MD, Rothman TP (1991) Time of origin of neurons in the murine enteric nervous system: sequence in relation to phenotype. J Comp Neurol 314:789-798.

Rothlein R, Springer TA (1986) The requirement for lymphocyte function-associated antigen 1 in homotypic leukocyte adhesion stimulated by phorbol ester. J Exp Med 163:1132-1149.

Satsangi J, Parkes M, Louis E, Hashimoto L, Kato N, Welsh K, Terwilliger JD, Lathrop GM, Bell JI, Jewell DP (1996) Two stage genomewide search in inflammatory bowel disease provides evidence for susceptibility loci on chromosomes 3, 7 and 12. Nat Genet 14:199-202.

Scherer SS (1997) The biology and pathobiology of Schwann cells. Curr Opin Neurol 10:386-397.

Seilheimer B, Schachner M (1988) Studies of adhesion molecules mediating interactions between cells of peripheral nervous system indicate a major role for L1 in mediating sensory neuron growth on schwann cells in culture. J Cell Biol 107:341-351.

Shimizu Y, Van Seventer GA, Horgan KJ, Shaw S (1990) Regulated expression and binding of three VLA $(\beta 1)$ integrin receptors on T cells. Nature 345:250-253.

Taniuchi M, Clark HB, Schweitzer JB, Johnson Jr EM (1988) Expression of nerve growth factor receptors by Schwann cells of axotomized peripheral nerves: ultrastructural location, suppression by axonal contact and binding properties. J Neurosci 8:664-681.

Vits L, Camp GV, Coucke P, Fransen E, De Boulle K, Reyniers E, Korn B, Poustka A, Wilson G, Schrander-Stumpel C, Winter RM, Schwartz C, Willems PJ (1994) MASA syndrome is due to mutations in the neural cell adhesion gene L1CAM. Nat Genet 7:408-413. 